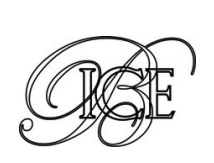

\author{
Antonio María Ávila Álvarez* \\ Miguel Ángel Díaz Mier**
}

\title{
REFLEXIONES SOBRE EL ACUERDO ADPIC
}

El Acuerdo sobre Derechos de Propiedad Intelectual (ADPIC) forma parte destacada importante de la Organización Mundial de Comercio (OMC). Conocerlo es una tarea especialmente necesaria. En este trabajo se estudian, en tres bloques, aspectos relativos a su nacimiento, su descripción y su evolución. Pretende ofrecer una primera visión de las cuestiones planteadas aportando los principales conceptos a analizar.

Palabras clave: acuerdos comerciales, derechos de propiedad intelectual.

Clasificación JEL: F13,O34.

\section{A la búsqueda de un acuerdo comercial en materia de propiedad intelectual}

En abril de 1994, en la ciudad de Marrakech, los representantes ministeriales de 123 países firmaron el Acta Final de la Ronda de Uruguay del GATT (Acuerdo General sobre Aranceles Aduaneros y Comercio), que se había lanzado en 1986. Igualmente, firmaron un Acuerdo por el que se establecía la Organización Mundial de Comercio (OMC). Los años siguientes contemplarían los necesarios procedimientos de ratificación de numerosos países, de manera que la OMC empezaría a funcionar en 1995. Hoy, a los países iniciales se han añadido otros que elevan a 164 el número total de miembros, entre los que se encuentran no solo las potencias comerciales

* Doctor en Derecho y Economía

** Profesor emérito Universidad de Alcalá.

Versión de noviembre de 2016. de este mundo sino también otros países calificados de «países en desarrollo».

Veinte años más tarde se ha llegado a una fecha significativa que permite, sin olvidar el pasado, la realización de valoraciones muy diversas sobre los resultados efectivos de la firma de los Acuerdos de 1994 y la evolución de los mismos, así como de la institución creada que constituye una de las grandes organizaciones del sistema de economía mundial. Recordaremos, en este trabajo de forma limitada, el Acta Final y su evolución, desde los estudios anteriores a 1986 hasta la actualidad. Nos parece necesario resaltar los antecedentes para entender mejor la indicada evolución.

Hemos de recordar que el Acta de Marrakech recoge tres anexos principales relativos a comercio de mercancías, definido como Anexo 1A, que, a su vez, incorpora doce acuerdos sobre materias específicas de ese comercio, el 1B relacionado con el comercio de servicios y $\triangleright$ 
finalmente el $1 \mathrm{C}$ que es el relativo a aspectos de los derechos de propiedad intelectual relacionados con el comercio (nos referiremos al mismo con sus siglas en español ADPIC o en inglés TRIPS. A ello hay que añadir -y conviene hacerlo por su implicación en el tema que examinamos- un «entendimiento» relativo a las normas y procedimientos por los que se rige la solución de diferencias, un mecanismo de examen de las políticas comerciales y algunos acuerdos plurilaterales que, a diferencia de todos los anteriores, no son aplicados por todos los miembros de la OMC, sino solo por los que lo ratifican.

Por adelantado, queremos expresar que en los anexos hemos recogido los títulos de las materias a que se refiere el ADPIC. También, y especialmente por razones académicas y profesionales, hemos de indicar que en la bibliografía hemos preferido limitar el número de obras citadas a una veintena, teniendo en cuenta que algunas de ellas recogen trabajos de autores muy notables en este campo o mejor dicho la pluralidad de temas que se recogen en la enumeración de los conceptos que han completado buen número de discusiones.

Hemos pretendido resumir en tres grandes apartados nuestras consideraciones. El primero de ellos, de forma muy sucinta, pretende mostrar el camino seguido por los países hasta llegar al Acuerdo de 1986. En el segundo, estudiamos las principales características de este ADPIC para finalizar con una exposición de las principales cuestiones que se han presentado en los decenios siguientes a 1995.

\section{Un repaso mínimo a la historia del ADPIC}

Cabe indicar que en los años de funcionamiento del Acuerdo General de Aranceles y
Comercio (desde 1948 hasta su integración en la OMC) no se encuentran normas específicas destinadas a la protección de los derechos de propiedad intelectual, si bien debe advertirse que algunos principios y ciertos mecanismos (la solución de diferencias, los procesos de toma de decisiones especialmente) se han incorporado hoy a la organización; la cual se centró básicamente en negociaciones arancelarias.

Pues bien, en la Ronda de negociación, conocida como la de Tokio, celebrada entre 1973 y 1979, se planteó por parte de los Estados Unidos la necesidad de llegar a algún tipo de acuerdo relativo al tratamiento del comercio de mercancías falsificadas, si bien la propuesta no prosperó y no encontramos referencias en los documentos que recogen los resultados de la Ronda indicada.

Los años siguientes, y especialmente el período 1982-1986, contemplarían iniciativas destinadas a ampliar los campos en los que el GATT podía actuar. En esta época sí se encuentran ya documentos relacionados con los problemas del comercio de mercancías falsificadas. Debe señalarse que la postura inicial de los Estados Unidos sería apoyada por un buen número de países desarrollados. Todos esos trabajos se tuvieron en cuenta en el lanzamiento de una nueva Ronda de negociaciones.

Las discusiones sobre las materias que debían incluirse en el proceso de negociación pondrían de relieve la insistencia de los Estados Unidos por incluir materias hasta entonces no abordadas explícitamente en el GATT, destacando así la petición de que se tratase el comercio de servicios y, ya en nuestro campo, la cuestión de los derechos de propiedad intelectual y su impacto en las relaciones comerciales. Esta posición no es compartida por varios países en desarrollo, encabezados $\triangleright$ 
por India y Brasil, que consideraban que el GATT no constituía el marco adecuado para negociar el tema, puesto que ya existían para ello organizaciones como la de Propiedad Intelectual dentro de Naciones Unidas.

Sin entrar en el detalle del proceso negociador, sí debemos indicar que finalmente en septiembre de 1986 se llegaría a un acuerdo sobre la forma de organizar las negociaciones y las materias que se incluirían en la Declaración de Punta del Este. Recordemos que, finalmente, se aceptaría la celebración de dos procesos paralelos: uno dedicado a materias que se referiría a comercio de mercancías y otro al comercio de servicios. Pues bien, entre las materias que se incluían en las indicadas de comercio de mercancías encontramos y es preciso resaltar lo siguiente:

- Aspectos de los derechos de propiedad intelectual relacionados con el comercio, incluido el comercio de mercancías falsificadas. Este título se concretó con el siguiente mandato.

- A fin de reducir las distorsiones del comercio internacional y los obstáculos del mismo -teniendo en cuenta la necesidad de fomentar una protección eficaz y adecuada de los derechos de propiedad intelectual y de velar para que las medidas y procedimientos destinados a hacer respetar dichos derechos no se conviertan a su vez en obstáculos al comercio legítimo- las negociaciones tendrán por finalidad clarificar las disposiciones del Acuerdo General y elaborar, según proceda, nuevas normas y disposiciones.

- Las negociaciones también tendrán por finalidad la elaboración de un marco multilateral de principio de normas y disciplina en relación con el comercio internacional de mercancías falsificadas, habida cuenta de la labor ya realizada en el GATT.
- Estas negociaciones se entenderán complementarias, sin perjuicio de otras iniciativas que puedan tomar en la Organización Mundial de la Propiedad Intelectual o en cualquier otro foro para tratar de resolver estas cuestiones.

Queremos destacar que, al igual que ocurría en otros campos de negociación, se había procurado hacer compatible las posiciones ya señaladas sobre el comercio de mercancías falsificadas, cuestión de interés especial para países desarrollados y de países que no consideraban el GATT como el marco para el tratamiento de los derechos de propiedad intelectual. Si bien quedaba aceptada la inclusión del tema en las negociaciones que se iniciarían en 1987, con un esquema de duración de cuatro años (que no se cumplirían). Nos parece necesario subrayar que, en los años siguientes y hasta la terminación de la Ronda Uruguay, el tema y su tratamiento siguieron las vicisitudes que experimentaría el proceso de negociación.

Nos referiremos, muy brevemente, a esta cuestión con un leve comentario a la documentación generada (más de setenta documentos de trabajo) que ocuparía la mayor extensión de nuestras reflexiones. Ahora bien, los trabajos de Roig y Warsserman, incluidos en el libro editado por Stewart y del funcionario de la OMC, A. Otten, que siguió, desde la Secretaría de la Institución, las cuestiones referentes al derecho de propiedad intelectual nos permiten destacar varias fases: a) hasta la celebración de la llamada «Midterm Review» en 1988; b) el período 1989-1990; c) los borradores de textos del período 1990-1991; d) el documento final.

La primera de las fases indicadas (celebrada en Montreal, en diciembre de 1988) se definiría por la necesidad de aclarar los conceptos de $\triangle$ 
la Declaración de Punta del Este, especialmente en nuestro campo. En definitiva, en varios temas (agricultura, servicios, derechos de propiedad intelectual) no se había podido conseguir acuerdos, que sí se habían logrado en otros. Por ello, destacamos los trabajos que resumen las posiciones de las distintas delegaciones en un documento elaborado por la Secretaría de la $\mathrm{OMC}$, que también recibiría una importante contribución de la Organización Mundial de la Propiedad Intelectual.

En abril de 1989 y para continuar la negociación que, como hemos indicado, no pudo llegar a un acuerdo en la reunión de Montreal, se produjo una aceleración de los temas pendientes, entre los que se incluía la negociación de la fórmula adoptada en Punta del Este. Para ello, se arbitró un procedimiento de trabajo, aprobado por los participantes, en una reunión a nivel ministerial, en la que se indicaría, como estructura para la continuación de los trabajos, una mayor precisión de la definición de la Declaración de Punta del Este. Así, se reconocía junto a la diversidad de opiniones de los países respecto al marco institucional, la posible aplicación de los principios básicos del GATT y los de acuerdos sobre propiedad intelectual de la dotación de medios efectivos y apropiados para el cumplimento de los derechos de propiedad intelectual, entre los que se encontrarían los procedimientos para la solución de diferencias. En nuestra opinión estos elementos se acercaban a los elementos institucionales que se estaban negociando sobre otras materias.

Las dos siguientes etapas que pueden englobarse en la denominación de borradores de textos, elaborados por un buen número de delegaciones, tanto de países desarrollados como de países en desarrollo, junto a unas «tablas sinópticas», obra de la Secretaría del
GATT, asignada a los trabajos, permitirían entrar en negociaciones específicas. Debe indicarse que las propuestas no tenían valor legal, pero preparaban el camino de la reunión de Bruselas que, de acuerdo con el esquema de la Declaración de Punta del Este, debía cerrar las negociaciones. Tal reunión se celebró en diciembre de 1990.

Debe destacarse que, por iniciativa del presidente del Grupo de Negociaciones, se presentaría para la indicada reunión de 1990 un texto ya en forma de articulado, y basado en la gran cantidad de trabajos a que hemos hecho referencia. Con frecuencia, este texto circularía, entre las distintas delegaciones, en forma de negociaciones informales. De esta forma, como indica Otten, el texto elaborado por el presidente del Grupo de Negociaciones y que no obligaba a ninguna delegación, contenía lo que puede describirse como un lenguaje común para varias partes del texto, pero no resolvía las diferencias respecto a la inclusión del tema en el GATT y tampoco quedaban resueltas otras cuestiones de importancia (la protección de los programas de ordenadores o las variedades de plantas, por ejemplo).

Como es bien conocido, la reunión de Bruselas con la que se debía finalizar la Ronda resultó fallida. No cabe duda que la cuestión de un acuerdo sobre agricultura -que no se conseguiría hasta varios años después y que todavía constituye materia de discusiones y trabajos dentro de la OMC- influiría de forma decisiva en este resultado.

Para terminar esta exposición, que pone de manifiesto el complejo proceso que se había seguido para llegar a «algo» (lógicamente en forma de acuerdo), hemos de referirnos a la fase final que se inició bajo la presidencia y la responsabilidad del director general del GATT, señor Dunkel, que elaboraría lo que, en el $D$ 
lenguaje de muchos participantes se conocería como «el ladrillo Dunkel» (por ser un compacto volumen de más de 400 páginas). Este documento se realizaba recogiendo los informes de los presidentes de los grupos de negociación (lo que significa que la distinción entre los que estudiaban temas de comercio de mercancías y comercio de servicios desaparecería) y consultas con delegaciones, destacando las aportaciones de ciertos grupos de negociación en los que ha de señalarse la fórmula del $10+10$, cifras que recogían diez países desarrollados y una cifra igual de países en desarrollo. El documento, conocido en diciembre de 1991, provocaría reacciones negativas por parte de algunos países, especialmente Estados Unidos, con presiones de su industria farmacéutica y de India.

Entre las cuestiones que quedaban pendientes de negociar (y que incluimos porque en los años siguientes al nacimiento de la OMC se volvieron a plantear) están: la situación de países que no proporcionaban una protección por medio de patentes a los inventos relativos a productos farmacéuticos, la necesidad de tomar en consideración los objetivos de políticas públicas y el calendario de entrada en vigor, cuestión especialmente compleja para los países que carecen de legislación respecto a los derechos de propiedad intelectual y que habían aceptado acuerdos internacionales como el Convenio para la protección de las obras literarias y artísticas.

$\mathrm{Ha}$ de recordarse que, finalmente y en especial, a consecuencia de las diferencias entre Estados Unidos y la Comunidad Europea, en materia agrícola, con prácticas condenadas por el GATT, se llegaría a un acuerdo para solucionarlas (Acuerdo de Blair House) que precipitaría el final de los trabajos de la Ronda y que, tras un breve período de negociación, conduciría al punto de partida de nuestro trabajo: el Acuerdo de Marrakech.

\section{Unas breves consideraciones sobre el ADPIC}

En el Anexo a nuestro trabajo, con objeto de facilitar el seguimiento del tema a posibles lectores y siguiendo el orden establecido en el texto final del acuerdo que ahora se denomina «Aspectos de los Derechos de Propiedad Intelectual relacionados con el Comercio», hemos recogido el índice del APIC, lo que permite marcar la diferencia con la definición que se habría adoptado en la Conferencia de Punta del Este. Igualmente, hacemos una referencia a los principios básicos que, en definitiva, han recogido posiciones concretas de los distintos países presentadas en el proceso de negociación. El ADPIC se articula a lo largo de 73 títulos que lógicamente no podemos reproducir.

Pues bien, para algunos autores como McGovern, la elaboración del ADPIC y del Acuerdo de Servicios, son el logro más notable del Tratado de Marrakech. Como hemos señalado, la presencia del GATT en estos dominios constituía una cuestión bien delicada por la existencia de una agencia especializada de las Naciones Unidas -la OMPI- y, además, existían convenios internacionales que habían de considerarse, si bien los mismos presentan varios inconvenientes: a) no todos los miembros del GATT, en 1999, habían firmado y ratificado muchos de esos convenios; b) distaban de proporcionar medios efectivos para resolver las diferencias comerciales; sancionar los incumplimientos y atender a las necesidades de los países en desarrollo.

Un examen somero de la parte I, relativa a disposiciones generales, nos lleva a la consideración elemental de que el ADPIC forma $\triangle$ 
parte de la familia de la OMC en la medida que incorpora los principios tradicionales de la OMC, heredados del GATT (el trato nacional y el de nación más favorecida), pero hay una matización, los miembros habrán de aplicar las disposiciones del acuerdo en el que se establece que, como mínimo, deben cumplir los criterios establecidos en una serie de convenios internacionales (el de París de Protección de la Propiedad Industrial y el de Berna para la Protección de Obras Literarias y Artísticas como más importantes), pero los miembros podrán prever en su legislación una protección mayor que la exigida en el acuerdo.

Para completar las obligaciones generales, que han planteado serias dificultades a los países en desarrollo que carecían de legislación nacional de protección de los derechos de propiedad intelectual, ha de hacerse una referencia al principio de transparencia que se refería a la publicación de leyes, reglamentos, resoluciones administrativas, decisiones jurisdiccionales y que formalmente se encuentran en la parte $\mathrm{V}$ del acuerdo, pero que nos parece adecuado incorporarlas a los principios antes indicados.

Los conceptos incluidos en las partes II, III y IV del acuerdo se referían por una parte a la existencia, alcance y ejercicio del derecho de propiedad intelectual, cuya simple enunciación pone de manifiesto que se trata de conceptos en discusión académica y profesional. Referirse a derechos de autor, indicaciones geográficas o patentes, lleva inmediatamente a realizar referencias a un buen número de obras de alcance y que han dado origen a una amplísima bibliografía.

La parte II es recogida por la Secretaría de la OMC bajo el título de normas de protección sustantivas y se refiere a ciertos conceptos que comentaremos brevemente.
1. Respecto a derechos de autor, el acuerdo señala que su protección se refiere a las expresiones y no a las ideas, procedimientos, métodos de operación o conceptos matemáticos. Para ello, el ADPIC señala que los miembros observarán las normas internacionales que se encuentran en modo primordial en el Tratado de Berna (1971) que, como anteriormente hemos dicho, se refiere a la protección de obras literarias y artísticas con la excepción de su artículo 6 bis relativo a derechos morales. Dentro de este concepto se incorpora la protección de programas de ordenador y recopilación de datos.

EI ADPIC, en lo que hace al concepto, proporciona a los autores de obras cinematográficas y programas de ordenador, como mínimo, el derecho de autorizar o prohibir el arrendamiento comercial al público de los originales o las copias de las copias amparadas por los derechos de autor. La protección se refiere también a los derechos de los artistas, intérpretes o ejecutantes, los derechos de registro y reproducción de música, programas radiofónicos y derechos en relación con películas y emisiones de televisión. La duración de la protección fue motivo de grandes discusiones en la etapa de negociación del acuerdo, pero al respecto se establece como norma un período de 50 años.

2. Entre los conceptos incluidos figuran las marcas de fábrica o de comercio (trademarks) definidos en el Convenio de París, y su acta de Estocolmo de 1967. El acuerdo especifica lo que constituye una marca «bien conocida». La protección se extiende al titular que gozará del derecho exclusivo de impedir que terceros utilicen, sin su consentimiento, signos idénticos o similares a los que sirvieron para registrar la marca. Se ofrece una protección, casi sin límite, al señalar que se puede renovar el registro de marca cada siete años. $\quad \square$ 
3. Con la denominación de indicaciones geográficas se busca la identificación de determinados productos como originarios del territorio de un miembro o de una localidad origen del mismo. El ADPID establece que los miembros no permitirían que se utilicen medios que en la presentación de un producto puedan inducir a error al público en cuanto al origen geográfico del mismo, y designar el registro de marcas que también puedan inducir a error. El acuerdo también prevé la realización de negociaciones destinadas a mejorar la protección de las denominaciones geográficas.

4. Respecto a dibujos y modelos industriales, el ADPIC prevé una protección para los mismos durante 10 años cuando sean nuevos u originales, los titulares de derechos estarán legitimados para impedir que terceros, sin su consentimiento, vendan, fabriquen o importen artículos que incorporen dibujos que sean copia del modelo protegido.

5. Sin duda, el aspecto de los derechos de propiedad intelectual al que el acuerdo dedica una mayor atención, como había ocurrido en el proceso negociador del mismo, es el de las patentes. De hecho, se dedican al tema ocho artículos, que se refieren a materias patentables que «podrán obtenerse por todas las invenciones, sean del producto o de procedimiento en todos los campos de la tecnología, siempre que sean nuevas,... una actividad inventiva y sean susceptibles de aplicación industrial». Con referencia a una cuestión, que más adelante ocupará buena parte de los trabajos de la OMC, los miembros podrán excluir de la patentibilidad a plantas y animales, si bien se preveía en 1995 que se «otorgará protección a las obtenciones de vegetales» mediante un sistema sui generis.

La duración de la patente se establece en 20 años y cubre «la fabricación, la utilización, la venta y la importancia del producto o el procedimiento». Se ha planteado como una cuestión compleja la protección de los derechos conferidos por una patente frente a terceros no autorizados.

6. La protección de los esquemas de trazado (topografías) a circuitos integrados (semiconductores) se había regulado en 1989 por el mal llamado Acuerdo de Washington. El ADPIC fortalece las disciplinas que en el mismo se habían constituido y establece una duración de 10 años. El ADPID señala como principio que «los miembros considerarán ilícitos determinados actos si se realizan sin la autorización del titular de derecho». Entre ellos figura de modo principal la importación, venta o distribución con fines comerciales de un esquema protegido.

7. El ADPID implementa el Acuerdo de París, tantas veces señalado, para que las normas de un miembro puedan buscar la garantía de una protección eficaz contra la competencia desleal. Así, señala que las personas físicas o jurídicas tendrán la posibilidad de impedir que la información que esté legitimada, bajo su control, se divulgue a terceros sin consentimiento.

La parte III del acuerdo se refiere a la observancia de los derechos de propiedad intelectual estableciendo como obligación general que los miembros del ADPID «se asegurarán de que en su legislación nacional se establezcan procedimientos de observancia de los derechos de propiedad intelectual», que permitan la adopción de medidas eficaces contra cualquier acción infractora de los mismos, incluidos en el acuerdo. En esta línea se hace una referencia a procedimientos y recursos civiles y administrativos, a procedimientos penales $y$, de modo especial, a medidas en frontera que puedan referirse a la suspensión del $\triangleright$ 
despacho de aduana por las autoridades aduaneras.

En una línea similar a la que hemos presentado, la parte IV del acuerdo se refiere a las disposiciones de los miembros para la adquisición y mantenimiento de los derechos de propiedad intelectual, que obligaría al establecimiento entre las normas reguladoras propias de cada país al respecto de procedimientos y trámites razonables. Cabe señalar aquí que el examen de tales normas se lleva a cabo de acuerdo con las características institucionales de la OMC a las que hace referencia las partes $\mathrm{V}$ y VII del acuerdo.

Así, en el orden institucional se establece un Consejo ADPIC, que depende directamente del Consejo General, que es el órgano de mayor nivel operativo integrado por representantes de todos los miembros y que se reúnen en los intervalos entre sesiones de la Conferencia Ministerial, que constituye el órgano de nivel máximo para adoptar decisiones y que ha de reunirse por lo menos una vez cada dos años. Hoy, en 2016, ha de señalarse que hasta la fecha se han celebrado diez conferencias, estando prevista la undécima para 2017. Estas conferencias llevan el nombre de la ciudad en que se celebraron.

Una forma de seguir los trabajos del ADPIC es acudir a la documentación generada sobre este acuerdo en la serie de conferencias, como tendremos ocasión de señalar. Bajo este órgano también pueden seguirse los trabajos del Consejo de los ADPID que tiene un nivel similar a los Consejos sobre acuerdos multilaterales (mercancías y servicios).

No obstante, hay una diferencia, como puede observarse, en los organigramas oficiales facilitados por la Secretaría de la OMC. En efecto, en estos dos acuerdos y sus órganos institucionales encontramos acuerdos específicos, lo que no es el caso del ADPID.
Finalmente, el ADPIC, en lo que se refiere a la solución de diferencias, será de aplicación al sistema establecido también en la Ronda de Uruguay. Un estudio de los casos planteados en el ámbito ADPIC pone de manifiesto que, desde 1996 hasta 2013, se han planteado 34 cuestiones. Su estudio permite conocer las materias que dentro del acuerdo han dado origen al funcionamiento del sistema. Podemos destacar los litigios planteados por Estados Unidos respecto a la protección mediante la patente de productos farmacéuticos y productos químicos para la agricultura establecidos por Pakistán e India o a las industrias relativas a los productos del tabaco establecidos por Australia y reclamados por Cuba, Honduras, Ucrania o República Dominicana.

\section{La evolución del ADPIC}

En este apartado pretendemos exponer de forma muy breve, y hasta cierto punto provisional, sin hacer referencia específica a normas, lo que en nuestra opinión, y de acuerdo con los trabajos de síntesis elaborados por la Secretaría de la OMC, serían los aspectos a considerar del período de 20 años transcurridos desde el nacimiento de la OMC y del ADPIC, que lógicamente siguen los avatares de la institución principal que se pone de manifiesto en las Conferencias Ministeriales.

Hemos de destacar, así, en primer lugar, el amplísimo número de documentos facilitados por los miembros en cumplimiento de las obligaciones de transparencia establecidas en el acuerdo. Se estima en más de 3.000 la cifra de las normas legales que los miembros han ido proporcionando. En segundo término, queremos destacar la mayor cooperación $D$ 
con instituciones internacionales, en especial los trabajos conjuntos con la OMPI que ha permitido proporcionar detalles sobre la estimación cuantitativa de los ingresos y pagos que se asocian en el comercio internacional o los derechos de propiedad intelectual. A su vez, cabe destacar la colaboración, en materia de salud pública, con la Organización Mundial de la Salud, y finalmente la participación del acuerdo en la creación de capacidad comercial mediante diversas fórmulas (la celebración de simposios), la denominada específicamente Ayuda para el Comercio destinada a los países en desarrollo.

Pero hemos de señalar varios temas y conceptos que, en varios casos, buscan la incorporación del ADPIC a materias que han ido surgiendo en el período indicado.

1. Destaquemos así, porque el texto del acuerdo lo indica, la cuestión de las denominaciones geográficas y su protección. En este orden ha de señalarse que, en concreto, desde la Conferencia de Doha (2001) se examinan dos cuestiones, la creación de un registro multilateral para los vinos y bebidas espirituosas y la extensión de un nivel más elevado de protección para productos distintos a los vinos. En estos temas se ha encargado la celebración de negociaciones al Consejo de los ADPIC, reunido en su sesión extraordinaria, como se estableció en la Conferencia.

Hoy encontramos, en ese orden, una propuesta muy detallada de la Unión Europea y otros miembros, pero otros no lo han estimado. La primera fórmula obligaría a una ampliación del acuerdo mediante un anexo y se referiría a un examen de formalidades, reservas, contenido, forma de registro y consecuencias del mismo. La otra no desearía que se modificase el acuerdo.

No es extraño que, en lo que se refiere al nivel más elevado de protección, se encuentren posiciones divergentes y pese a los documentos de síntesis, que han ido elaborando los presidentes de los grupos que han abordado la cuestión, aún no se ha hallado la fórmula de acuerdo.

2. Un segundo tema que se ha abordado en el período examinado tiene su origen en el acuerdo, en cuanto se presenta el tema de la patentabilidad o no de las invenciones relacionadas con las plantas y los animales y la protección de las obtenciones vegetales. Pero ya en la indicada Conferencia de Doha se encomienda al Consejo de los ADPIC que lleve a cabo un examen de la relación que pudiera existir entre el acuerdo que hemos presentado y un Convenio de las Naciones Unidas sobre la diversidad biológica, la protección de los conocimientos tradicionales y el folklore.

En estos órdenes encontramos hoy una documentación específica preparada técnicamente por la Secretaría de la OMC aunque, en la media en que seguimos el tema, no parece que se haya llegado a algún tipo de recomendación.

3. Otra cuestión de actualidad innegable, y en la que la OMC y especialmente el ADPIC tiene un papel que jugar, son los temas relacionados con el cambio climático, especialmente el Convenio Marco de las Naciones Unidas sobre el Cambio Climático celebrado en 1992 y el reciente Acuerdo de París de 2016.

En efecto, la búsqueda de tecnologías «verdes», es decir, las que aplican prácticas ambientalmente sanas, ha incidido en el ADPIC. En la medida en que este acuerdo forma parte del sistema institucional mundial, está inmerso en la promoción de la innovación tecnológica, su transferencia y difusión.

El tema se ha planteado en diversas reuniones del Grupo del ADPIC, a la vez que la Secretaría de la OMC ha preparado una interesante documentación sobre la pertinencia $\triangleright$ 
de las disposiciones del ADPIC y el indicado Convenio de Naciones Unidas. En la actualidad no hemos hallado información sobre la compatibilidad o no de los derechos de propiedad intelectual con el convenio indicado, aunque se puede conocer posiciones de algunos países reflejadas en las Actas del Consejo ADPIC, en las que las delegaciones han puesto de relieve contribuciones de centros científicos y de universidades de Estados Unidos y Suiza especialmente.

4. Posiblemente una de las materias que han sido objeto de mayores trabajos en el período es la relativa a la labor del ADPIC en materia de salud pública, sobre la que como hemos resaltado hay formas de cooperación con la OMS (Organización Mundial de la Salud). De hecho, ya desde 2001 (Conferencia de Doha) se ha tratado el tema del equilibrio entre los avances científicos en materia de productos farmacéuticos y la patentibilidad de todos los productos con las medidas que, atribuidas a los países, puedan adoptar para defender la salud pública.

El propio acuerdo, como hemos indicado anteriormente, no concede derechos de patente sin límites. Especialmente hay que referirse a la necesidad de que el titular de una patente divulgue las especificaciones del producto patentado. Buen número de países en desarrollo, en que se había desarrollado y no tenían industria farmacéutica, se encuentran con los problemas de precios elevados para los productos patentados.

Se plantean, al respecto, las licencias obligatorias (compulsory licences), que son los permisos que un país (en este caso en desarrollo) concede para producir un producto patentado (y que, por lo tanto, según una interpretación in extenso de la empresa que un país desarrollado obligaría a los importadores a aceptar).
En definitiva, se trataría de buscar el equilibrio, en este caso, de un difícil equilibrio.

También se han planteado casos de importaciones paralelas que se entienden como importaciones de productos comercializados por el titular de la patente en un país e importados en otro sin la aprobación del titular de la patente.

Pues bien, en la Conferencia de Doha se adoptaría una Declaración que se centraba en que el Acuerdo sobre los Derechos de Propiedad Intelectual no debían impedir que los miembros adoptasen medidas con objeto de proteger la salud pública. En este sentido se subrayaría la capacidad de las partes para el uso de licencias obligatorias y de importaciones paralelas y especialmente la importación de medicamentos genéricos más baratos. Más tarde se establecía un sistema de notificación para los países que, como importadores o exportadores, hagan uso de las licencias obligatorias.

5. Probablemente, las materias que han tenido más incidencia en la OMC, y por tanto también en el ADPIC, son las relacionadas con la transferencia de tecnología. Debe recordarse que para que muchos países en desarrollo aceptasen el ADPIC, buscaron asociarlo a tal transferencia y así se recoge en el resumen de principios que hemos incluido en el Anexo. Las negociaciones incluidas en el Programa de Doha para el Desarrollo o las actividades para la creación de capacidad comercial de la OMC forman parte principal de este concepto.

El acuerdo que hemos presentado constituye un buen ejemplo de evolución del sistema comercial multilateral basado en la OMC. Su análisis puede realizarse desde muchos puntos de vista: jurídico, de relaciones internacionales, económicos, etcétera, pero tras $D$ 
20 años de funcionamiento, con todas sus complejidades y problemas, ha de repetirse la afortunada frase de «si no existiese habría que inventarlo». Nos parece algo que no se debe olvidar, máxime cuando se sabe que el comercio legal (dado que en esta materia hay mucha piratería que puede incluso estimarse en la misma cifra que en el comercio legal) superan los 30.000 millones de dólares anuales. 


\section{ANEXO RECORDATORIO DEL ÍNDICE DEL ADPIC Y SUS PRINCIPIOS}

PARTE I. Disposiciones generales y principios básicos

PARTE II. Normas relativas a la existencia, alcance y ejercicio de los derechos de propiedad intelectual

1. Derecho de autor y derechos conexos

2. Marcas de fábricas o de comercio

3. Indicaciones geográficas

4. Dibujos y modelos industriales

5. Patentes

6. Esquemas de trazado (topografías) de los circuitos integrados

7. Protección de la información no divulgada

8. Control de las prácticas anticompetitivas de las licencias contractuales

PARTE III. Observancia de los derechos de propiedad intelectual
1. Obligaciones generales
2. Procedimientos y recursos civiles y administrativos
3. Medidas provisionales
4. Prescripciones especiales relacionadas con las medidas en frontera
5. Procedimientos penales

PARTE IV. Adquisición y mantenimiento de los derechos de propiedad intelectual y procedimientos contradictorios relacionados

PARTE V. Prevención y solución de diferencias

PARTE VI. Disposiciones transitorias

PARTE VII. Disposiciones institucionales. Disposiciones finales

Fuente: elaboración propia.

\section{ANEXO RELATIVO A LOS PRINCIPIOS ACORDADOS EN LAS NEGOCIACIONES}

a) Aplicabilidad de los principios GATT a los acuerdos internacionales pertinentes en materia de propiedad intelectual. En especial el acuerdo señala los derechos de propiedad intelectual relacionados con el comercio, así como la provisión de medios eficaces para hacer respetar los derechos de propiedad intelectual relacionados con el comercio, y en especial la solución de diferencias entre Gobiernos

b) Aplicación al comercio internacional de marcas falsificadas

c) Reconocimiento que los derechos de propiedad intelectual se vean incluidos en un objetivo de política general pública

d) Han de tener en cuenta las necesidades especiales de los países en desarrollo

Fuente: elaboración propia. 